\title{
Paraneoplastic Filiform Hyperkeratosis and Immunoglobulin-Associated Vasculitis in Myeloma Progression: A Case Report
}

\author{
Mathias Oymanns $^{a} \quad$ Mehmet Baltaci $^{b} \quad$ Aliyah Bellm $^{c} \quad$ Chalid Assafa $^{a}$ \\ aDepartment of Dermatology, HELIOS Klinikum Krefeld, Krefeld, Germany; ${ }^{b}$ Department of \\ Dermatology, HELIOS Klinikum Duisburg, Duisburg, Germany; 'Department of Pediatrics, \\ HELIOS Klinikum Krefeld, Krefeld, Germany
}

\section{Keywords}

Case report · Paraneoplasia · Filiform hyperkeratosis · Immunoglobulin-associated vasculitis - Myeloma

\section{Abstract}

Multiple myeloma is a lymphoproliferative disease, which rarely presents with skin involvement or associated symptoms. Better awareness of these dermatological presentations is required for early diagnosis and to guide the patient towards appropriate therapy. We report on a patient with diffuse filiform hyperkeratosis and immunoglobulin-associated vasculitis in a severe progression of a known myeloma.

\section{Case Report}

A 58-year-old man presented to the hospital with spiky skin alterations over the entire body. On palpation, the spikes were hard, like touching a rasp (shown in Fig. 1, 2). These symptoms began 2 weeks before, and in addition, the patient noticed cutaneous bleedings on his hands and feet (shown in Fig. 3, 4). Further clinical examination showed no lymphadenopathy. Medical history included an earlier diagnosis of multiple myeloma, Durie-Salmon stage I, currently under a watch-and-wait approach. 


\begin{tabular}{|c|c|c|}
\hline \multirow[b]{2}{*}{ Case Reports } & \multicolumn{2}{|c|}{ Case Ren Dermatol 2021.13.563-567 } \\
\hline & DOl. $101159 / 000518420$ & (c) 2021 The Author(s). Published by S. Karger AG, Basel \\
\hline
\end{tabular}

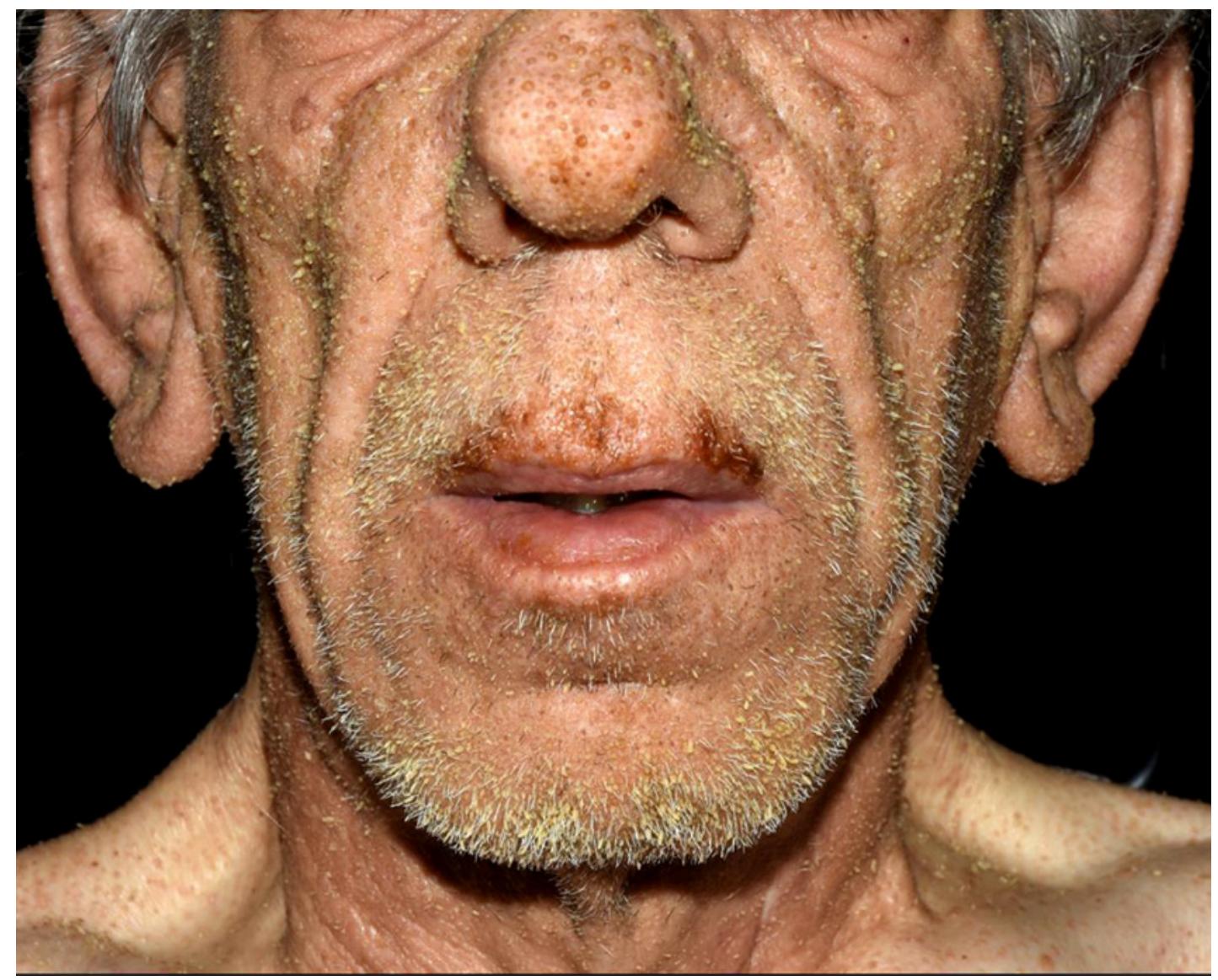

Fig. 1. Facial filiform hyperkeratosis.

Fig. 2. Filiform hyperkeratosis on the body.

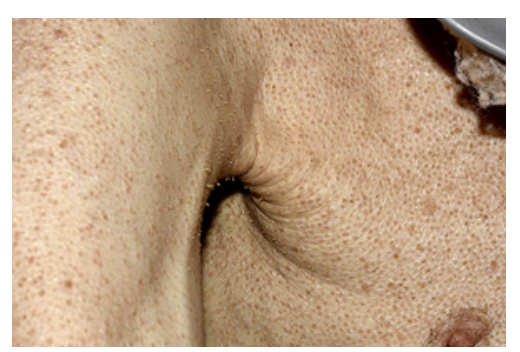

Blood tests revealed hypochromic, microcytic anaemia and an elevated CRP with a serum concentration of $87.7 \mathrm{mg} / \mathrm{L}$ (normal $<5 \mathrm{mg} / \mathrm{L}$ ). ESR was $94 \mathrm{~mm} / \mathrm{h}(<20 \mathrm{~mm} / \mathrm{h}$ ), and serum protein electrophoresis revealed a gamma peak of 59.4\% (9.2-19.4\%). Serum IgG was elevated at 7,070 mg/dL (700-1,600 mg/dL), and kappa/lambda ratio was 27.1 (0.26-1.65). Free serum kappa light chains were also elevated at $664 \mathrm{mg} / \mathrm{L}$ (3.3-19.4 mg/L).

Whole-body CT scan showed multiple osteolytic lesions throughout the skeleton (shown in Fig. 5). Histopathologic evaluation of a skin biopsy of the face revealed ectatic hair follicles, perifolliculitis, and follicular and non-follicular hyperkeratosis (shown in Fig. 6). In addition, a skin biopsy of the dorsal hand showed occlusion of the ectatic superficial dermal vascular plexus by PAS-positive hyaline material and showed signs of leukocytoclastic vasculitis (shown in Fig. 7,8), possibly consistent with immunoglobulin-associated vasculitis. 
Fig. 3. Purpura on the hands.

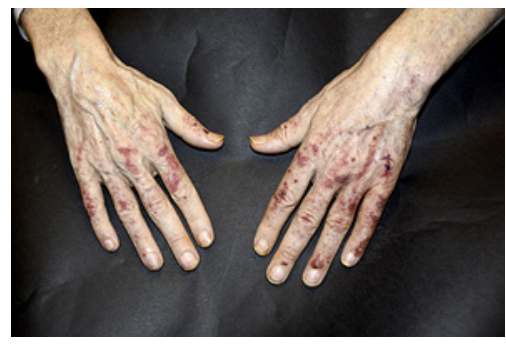

Fig. 4. Purpura on the feet.

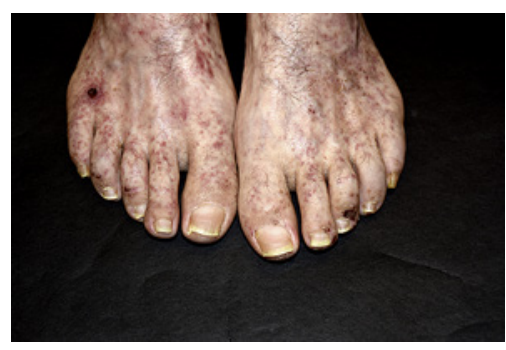

Fig. 5. Axial CT scan with multiple osteolytic lesions.

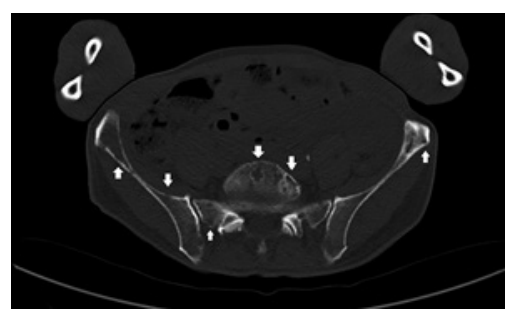

Fig. 6. HE stain, $\times 50$ with ectatic follicle and hyperkeratosis.

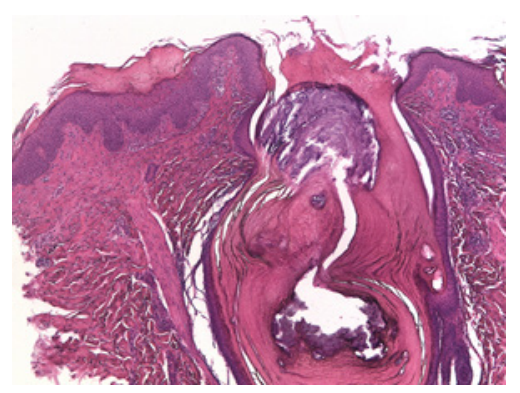

\section{Discussion}

Multiple myeloma is a disorder of haemoblastosis with neoplastic proliferation of immunoglobulin-producing plasma cells. Typically, the symptoms are caused by infiltration of plasma cells into the skeleton or due to a kidney damage related to this disease. Nevertheless, the disease can also present with skin symptoms as seen in our case. These skin symptoms are rare, mostly occur in late stages, and are a clinical sign of exacerbation/progression of the myeloma. Other specific skin lesions are very rare and include cutaneous or mucosal extramedullary plasmocytomas. Further skin symptoms encompass a broad spectrum of pathologies and include hyperkeratotic spikes, papules (e.g., sweet syndrome), ulcers (e.g., pyoderma gangrenosum), and vasculitic and purpuritic changes caused by amyloidosis or vessel occlusion $[1,2]$. Since skin 
Fig. 7. HE stain, $\times 100$ : occlusion of superficial dermal vessels.

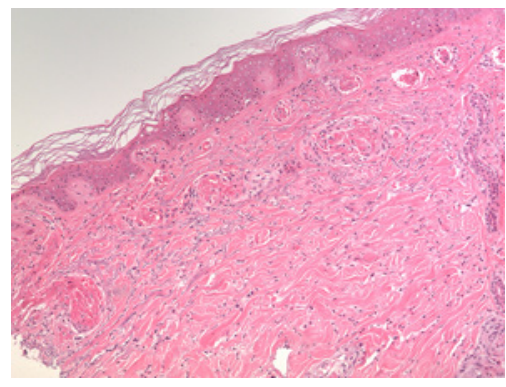

Fig. 8. PAS stain, $\times 400$ : homogeneous PAS-positive intraluminal material.

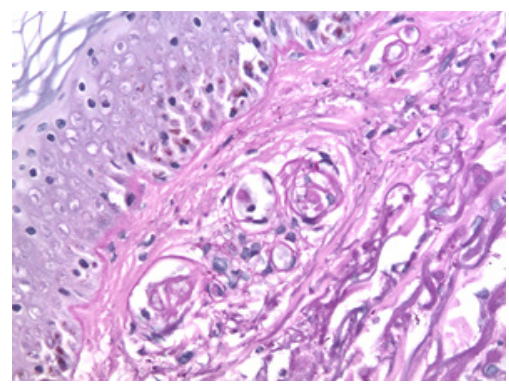

lesions may be the first sign of myeloma, it is important for clinicians to be aware of cutaneous paraneoplastic symptoms to aid in early diagnosis, thus enabling timely and adequate treatment.

Filiform hyperkeratosis in particular is characteristic of myeloma and should prompt further investigation. The pathogenesis of these skin findings in myeloma is not completely elucidated; however, precipitation of the monoclonal paraproteins in the skin and its blood vessels may play an important role [3-5]. Multiple case reports demonstrate filiform hyperkeratosis in myeloma with kappa and lambda light chains in IgG myeloma [4, 6-8]. It remains unclear if filiform hyperkeratosis is restricted to IgG myeloma and which myeloma patients are affected; however, the degree of paraproteinaemia may be critical for its development. We propose this thesis because of the extent of severe exacerbation in our patient.

Vasculitic changes in paraproteinaemia are usually on acral areas. A link to cold exposure could not be reported by our patient. A Raynaud phenomenon, a livedo racemosa, or acrocyanosis could not be observed, although ulceration has been present. In our laboratory, the cryoglobulins were excluded. Besides paraproteinaemic aetiology - as in our case the IgG myeloma - lympho- or myeloproliferative disorders, hepatitis $\mathrm{C}$, and autoimmune diseases should be ruled out as differential diagnoses [9]. In synopsis of the clinical, histological, and oncological aspects in our patient, we hypothesized that the vasculitis is provoked by immunoglobulins due to the IgG myeloma.

In summary, the cutaneous findings led to the diagnosis of a paraneoplastic phenomenon secondary to severe progression of the patient's myeloma, now Durie-Salmon stage III. Based on the staging and the patient's age, he was referred to a haematology-oncology centre for autologous stem cell transplantation.

\section{Statement of Ethics}

Written informed consent was obtained from the patient for publication of this case report and the accompanying images. The research was conducted ethically in accordance with the World Medical Association Declaration of Helsinki. 


\section{Conflict of Interest Statement}

The authors have no conflicts of interest to declare.

\section{Funding Sources}

None.

\section{Author Contributions}

M.O. was active in patient care and wrote the manuscript. A.B. corrected the manuscript as a native speaker. M.B. contributed to the histopathology, and C.A. supervised the case and edited the final version of the manuscript. All authors provided critical feedback and contributed to the final version of the manuscript.

\section{Data Availability Statement}

All data generated or analysed during this study are included in this article and its online supplementary material files (for all online suppl. material, see www.karger.com/ doi/10.1159/000518420). Further enquiries can be directed to the corresponding author.

\section{References}

1 Bayer-Garner IB, Smoller BR. The spectrum of cutaneous disease in multiple myeloma. J Am Acad Dermatol. 2003;48:497-507.

2 Daoud MS, Lust JA, Kyle RA, Pittelkow MR. Monoclonal gammopathies and associated skin disorders. J Am Acad Dermatol. 1999;40:507-8.

3 Bork K, Böckers M, Pfeifle J. Pathogenesis of paraneoplastic follicular hyperkeratotic spicules in multiple myeloma. Follicular and epidermal accumulation of IgG dysprotein and cryoglobulin. Arch Dermatol. 1990; 126(4):509.

4 Satta R, Casu G, Dore F, Longinotti M, Cottoni F. Follicular spicules and multiple ulcers: cutaneous manifestations of multiple myeloma. J Am Acad Dermatol. 2003;49:736-40.

5 Hosler GA, Weibel L, Wang RC. The cause of follicular spicules in multiple myeloma. JAMA Dermatol. 2015; 151:457-8.

6 Requena L, Sarasa JL, Ortiz Masllorens F, Martín L, Piqué E, Olivares M, et al. Follicular spicules of the nose: a peculiar cutaneous manifestation of multiple myeloma with cryoglobulinemia. J Am Acad Dermatol. 1995;32: 834-9.

7 Tay LK, Lim FL, Ng HJ, Lee HY, Pang SM, Thirumoorthy T. Cutaneous follicular hyperkeratotic spicules: the first clinical sign of multiple myeloma progression or relapse. Int J Dermatol. 2010;49:934-6.

8 Smith MP, Manabat-Hidalgo C. Follicular spicules of multiple myeloma. Dermatol Online J. 2019;25(10): 13030/qt5bp8s5nn.

9 Gause A, Lamprecht P. Die kryoglobulinämische vaskulitis. Internist. 2003;44(2):165-74.

\section{Karger'}

